\title{
Compensation continues rise
}

Stacy Lawrence

The divide between compensation for R\&D staff in industry and academia continues to widen. Median average salary in a private organization is now $\$ 30,000$ more than in academia. Despite the economic downturn, life sciences execs as a whole are targeting a $9 \%$ average

\section{Median salaries in industry and academia}

For those working in R\&D, industry remains more competitive in terms of salaries.

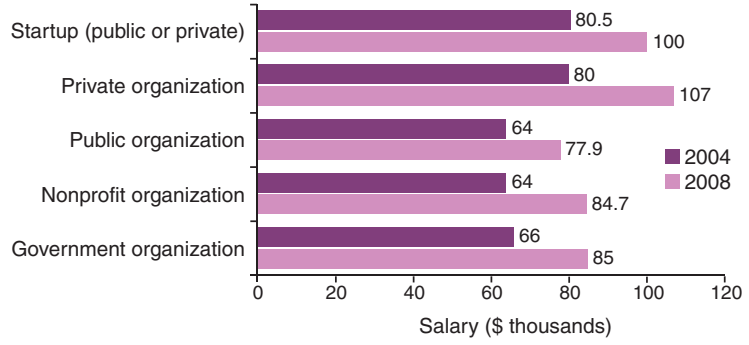

Source: The Scientist 2008 Life Science Salary Survey

\section{Life sciences executive perks}

The top five titles hold on average about $13 \%$ of company shares; almost three-quarters of CEOs have a golden parachute.

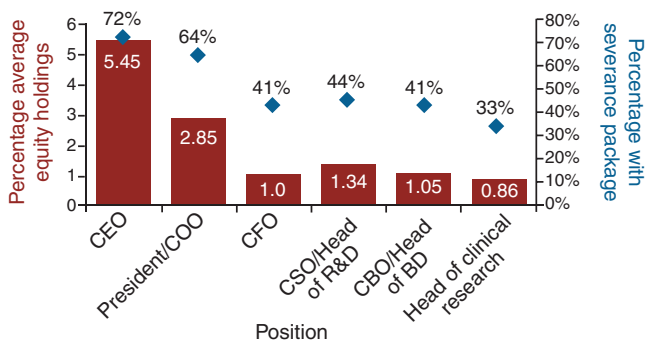

Data include medical device companies. CSO, chief scientific officer; CBO, chief business officer; BD, business development. Source: WilmerHale, Ernst \& Young, J. Robert Scott

\section{Biotech employees, revenue and R\&D}

\begin{tabular}{|c|c|c|c|c|c|c|c|c|}
\hline Company name & 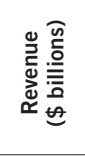 & $\begin{array}{l}\text { ఖ } \\
\frac{0}{0} \\
\frac{0}{E}\end{array}$ & 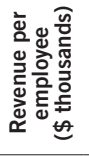 & 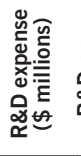 & 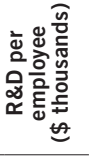 & 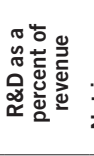 & 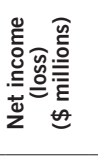 & 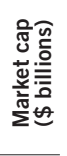 \\
\hline Amgen & 14,771 & 17,500 & 844 & 3,266 & 187 & $22 \%$ & 3,166 & 47 \\
\hline Genentech & 9,443 & 11,174 & 845 & 2,523 & 226 & $27 \%$ & 2,769 & 78 \\
\hline Gilead Sciences & 4,230 & 2,979 & 1,420 & 591 & 198 & $14 \%$ & 1,615 & 8 \\
\hline Genzyme & 3,784 & 10,000 & 378 & 738 & 74 & $19 \%$ & 480 & 9 \\
\hline Biogen Idec & 3,172 & 4,300 & 738 & 925 & 215 & $29 \%$ & 638 & 19 \\
\hline $\begin{array}{l}\text { Applied Biosystems } \\
\text { Group - Applera }\end{array}$ & 2,089 & 5,000 & 418 & 204 & 41 & $10 \%$ & 171 & 5 \\
\hline Cephalon & 1,727 & 2,796 & 618 & 369 & 132 & $21 \%$ & (192) & 4 \\
\hline Celgene & 1,406 & 1,685 & 834 & 399 & 237 & $28 \%$ & 226 & 25 \\
\hline Average & & & 762 & & 164 & $21 \%$ & & \\
\hline
\end{tabular}

Data for FY 07. Market cap as of year end 2007. Source: Reuter Global Fundamentals via FactSet Research Systems, Nature Biotechnology. increase in their cash compensation in 2008; CEOs are even more aggressive, aiming for an average $12 \%$ rise to $\sim \$ 400,000$. Top execs based in New England make the most on average; those in the Midwest receive $20-33 \%$ less.

\section{Life sciences executives' cash compensation}

Pay increased $\sim 5 \%$ last year, with CEO salaries up from $\$ 350,000$ in 2007 to almost $\$ 400,000$.

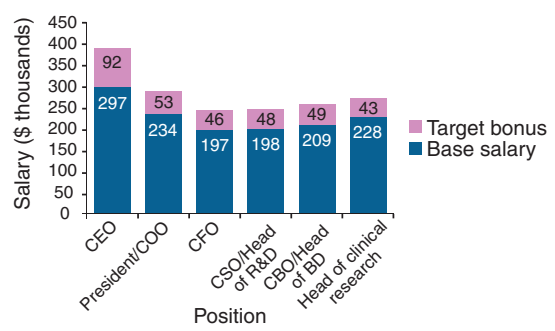

Data include medical device companies. Source: WilmerHale, Ernst \& Young, J. Robert Scott

\section{Employment at public biotech companies by region}

The sector's number of employees $(200,000)$ grew relatively little, although Europe contributed $2 \%$ more to the total than last year, whereas US employee number declined slightly.

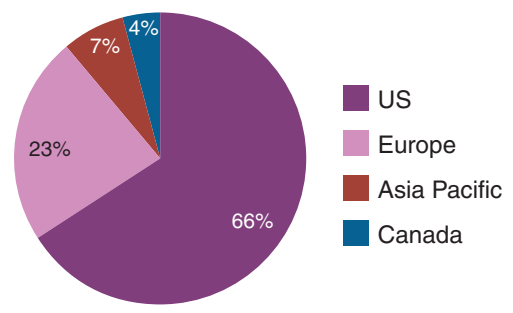

Based on 2007 data. Source: Ernst \& Young

Cash compensation by US region

\begin{tabular}{|c|c|c|c|c|c|c|}
\hline Region & 웜 & 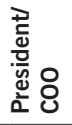 & 윤 & 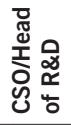 & 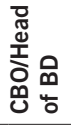 & 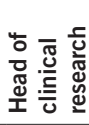 \\
\hline New England & $\$ 422$ & NA & $\$ 268$ & $\$ 287$ & $\$ 293$ & $\$ 325$ \\
\hline California & $\$ 404$ & $\$ 316$ & $\$ 241$ & $\$ 266$ & $\$ 279$ & $\$ 293$ \\
\hline Mid-Atlantic & $\$ 376$ & $\$ 272$ & $\$ 260$ & $\$ 244$ & $\$ 257$ & $\$ 301$ \\
\hline South & $\$ 337$ & $\$ 276$ & $\$ 211$ & $\$ 227$ & NA & NA \\
\hline Midwest & $\$ 267$ & $\$ 182$ & $\$ 214$ & $\$ 177$ & NA & NA \\
\hline West (excluding & NA & NA & $\$ 248$ & NA & NA & NA \\
\hline
\end{tabular}

California)

Data for 2008. In thousands, includes base salary and 2008 target bonus. Data include medical device companies. CSO, chief scientific officer; $\mathrm{CBO}$, chief business officer; BD, business development; NA, not available. Source: WilmerHale, Ernst \& Young, J. Robert Scott 